\title{
Les Barrières à la Pratique des Sages-Femmes au Canada
}

\author{
Isabelle Vézina, BSI \\ Robyn Gorham, PhD cand. \\ Elena Hunt, PhD \\ Université Laurentienne, Canada
}

URL:http://dx.doi.org/10.19044/esj.2019.v15n27p1

\section{Résumé}

Les barrières à la pratique actuelle des sages-femmes au Canada sont reliées à la perception des pourvoyeurs de soins et des clientes, au manque de ressources matérielles et humaines, aux réticences de la part du personnel médical et à un manque de reconnaissance de l'activité des sages-femmes et de leurs compétences. Malgré que la plupart des accouchements se déroulent sans complications graves, les grossesses à faibles risques sont souvent prises en charge par des médecins, plutôt que par des sages-femmes. Au Québec, de longues listes d'attente empêchent les femmes d'avoir recours à une sagefemme car ce service est peu développé, même inexistant par endroit, et ce, malgré la satisfaction des soins reportée par les parturientes. Les programmes de formation de sages-femmes sont contingentés. Au niveau mondial, l'accès à la pratique des sages-femmes est restreint en raison d'un manque de financement, bien que la pratique des sages-femmes génère moins de coût que la pratique des autres praticiens. De plus, les obstétriciens ne sont pas ouverts à l'idée de perdre les cas les moins compliqués, pouvant être confiés aux sages-femmes. En tout et compte tenu des difficultés budgétaires actuelles, les gouvernements auraient avantage à investir dans cette profession qui, de toute évidence, démontre clairement son efficacité et offre des soins de qualité.

Mots-clés: Sages-femmes, Barrières à la pratique, Reconnaissances, Services, Dépenses budgétaires 


\title{
Barriers to Practice of Midwives in Canada
}

\author{
Isabelle Vézina, BSI \\ Robyn Gorham, PhD cand. \\ Elena Hunt, PhD \\ Universite Laurentienne, Canada
}

\begin{abstract}
Barriers to the current practice of midwives in Canada relate to the perception of clients and care providers, the lack of financial and physical resources, the reluctance on the part of other medical specialists and a lack of recognition of midwives professional activity and their skills. Although deliveries without serious complications are the vast majority of births, lowrisk pregnancies are often supported by physicians, rather than by midwives. In Quebec, long waiting lists prevent women from resorting to a midwife because, often, this service is simply not present, despite the satisfaction with care extended by the mothers. Training programs for midwives have limited contingency. At the global level, access to the practice of midwives is restricted due to lack of funding, although the practice of midwives generates less cost than other practitioners'. In addition, obstetricians are not open to the idea of losing less complicated cases, handled by midwives. However, in light of the current budgetary constraints, Governments would benefit from investing in the profession of midwives which clearly demonstrates its effectiveness in the delivery of quality care.
\end{abstract}

Keywords: Midwives, Barriers to practice, Recognition, Services, Budgetary constraints

\section{Introduction}

La profession de sage-femme est sans doute la profession la plus ancienne, $<<[\ldots]$ tout simplement parce que les femmes accouchent depuis le début des temps et que l'on a des preuves à l'effet que lors de l'accouchement, les femmes ont toujours été aidées par d'autres femmes >> (Mélanson, 1999). C'est encore le cas à l'heure actuelle en Hollande, où $80 \%$ des naissances sont toujours assistées par des sages-femmes ou en Grande-Bretagne, dont la majorité des naissances sont intégrées sous la responsabilité des sages-femmes (McCracken, 2015). Au Canada, cette mentalité a changé après la deuxième guerre mondiale, soit à l'ère technologique. Les accouchements furent, pour 
des raisons sociales, politiques et économiques, transmises entre les mains des médecins et gérés dans les hôpitaux. La pratique des sages-femmes est alors devenue marginalisée pendant de nombreuses années : permise, mais noncouverte par l'assurance médicale provinciale. Les parturientes qui désiraient employer les services d'une sage-femme devaient débourser les frais de leur poche. Aujourd'hui, la profession est en expansion et développement, mais le progrès est modeste et inégalement réparti à travers le pays.

Après plus d'un demi-siècle, une question se pose toujours par rapport à cet état des choses, à savoir : Quelles sont les barrières à la pratique actuelle des sages-femmes au Canada?. Que ce soit relié à une question de perception, de changement, de manque de ressources humaines et matérielles, de réticences de la part des autres spécialistes ou d'un manque de reconnaissance, la profession des sages-femmes demeure restreinte au Canada.

\section{But de l'étude}

Cet article discute la politique de l'accouchement au Canada, plus particulièrement au Québec. C'est une prise de position vis-à-vis 'l'affaire' d'être naît, plus que rentable pour certains professionnels de la santé, qui en gardent le monopole. L'accouchement, n'étant pas une maladie, doit conserver son statut de processus physiologique naturel.

Méthode

Une recension des écrits et des politiques de l'obstétrique a été menée sur les bases de données de Santé Canada et CINAHL (Cumulative Index to Nursing and Allied Health Literature), afin d'identifier l'organisation des naissances et les barrières à la pratique des sages-femmes au Canada. Peu d'étude ont été localisés, probablement en raison du fait que la pratique des sages-femmes est gérée par les ordres professionnels provinciaux et par les politiques fédérales; ainsi, les recherches existantes relèvent plutôt de la satisfaction des parturientes et moins des barrières à la pratique des sagesfemmes. Les écrits recensés sont mentionnés dans la liste des références, ayant généré les résultats suivants.

Résultats et discussion

\section{Transformation des soins}

Les accouchements sans complications graves constituent 65 à $90 \%$ des cas au Québec (Dessureault, 2015). Ces grossesses, à faibles risques, pourraient très bien être prises en charge par des sages-femmes plutôt que par des médecins. Rappelons-nous qu'il n'y a pas moins de 100 ans, la grossesse et l'accouchement étaient vus comme des évènements normaux et non des 'maladies' comme ils sont considérés toujours, par endroit. Les obstétriciens, étant également des chirurgiens, pratiquent de plus en plus d'interventions durant les accouchements (déclenchement du travail, épisiotomie, césarienne, 
etc...). Ces interventions sont parfois non-nécessaires, ce à quoi les sagesfemmes n'adhèrent pas. Elles peuvent entraîner des conséquences négatives pour les familles, autant sur le plan physique que psychosocial.

En l'an 2000, 52\% des Québécoises ont eu recours aux anesthésiants durant leurs accouchements. Seulement 16\% des Québécoises accouchant par voie vaginale en hôpital n'ont pas eu recours à aucune anesthésie (Dessureault, 2015). L'anesthésie est administrée dans le but de soulager la douleur de la mère durant l'accouchement, alors qu'elle peut avoir des effets pervers. La mère dépend entièrement du corps médical pour savoir quand pousser, elle vit donc un accouchement passif. Les anesthésiants inhibent l'effet des endorphines et de l'ocytocine durant l'accouchement, hormones sécrétées naturellement par la femme durant l'accouchement. Les endorphines profitent au bébé et à la mère afin qu'elle puisse mieux gérer sa douleur et l'ocytocine régule la température du nouveau-né et est favorable à l'allaitement. La biologie humaine a donc sa propre solution aux souffrances de l'accouchement, mais ces hormones sont beaucoup moins sollicitées si on intervient artificiellement (Dessureault, 2015). Dans le but d'accélérer l'accouchement, le personnel médical utilise fréquemment le déclenchement du travail, par rupture artificielle des eaux ou en administrant des hormones, soit pour une naissance sur cinq (Santé Canada, 2008). Cette technique augmente l'intensité des contractions, donc de la douleur pour la mère, pouvant ainsi réduire le taux d'oxygénation du bébé. D’autant plus souffrantes, les femmes recourent souvent à l'épidurale dans ces situations, ce qui augmente les risques pour le nouveau-né et force le personnel médical à utiliser des moyens mécaniques et chirurgicaux pour accélérer la naissance. Le nombre de naissances autrefois réparti aléatoirement est aujourd'hui plus bas durant les fins de semaine et congés fériés, un signe que les accouchements pourraient être déclenchés pour des raisons d'horaires du personnel (Dessureault, 2015).

La pratique de la césarienne est en hausse depuis les années 60, elle a passé de 5 à 25\% (Dessureault, 2015) malgré le fait qu'il s'agit souvent d'une intervention médicale invasive non-nécessaire. Bien qu'il n'y ait plus de risque mortel pour les femmes de nos jours lors d'une césarienne, la douleur post-opératoire, les hémorragies, les infections et les ruptures utérines sont des risques présents.

$<<$ La peur de souffrir et de l'incontrôlable s'inscrit dans une société de consommation où tout se règle rapidement et où souffrir est inacceptable. Sans compter les risques innombrables associés à l'absence de la technologie dans une réalité où le taux de naissances est extrêmement bas et où il existe donc une certaine pression pour mettre au monde un enfant parfait. Les infrastructures en place, les modes de pensée dominants et l'état d'esprit de la femme pendant 
l'accouchement contraignent donc fortement la liberté de choisir le déroulement de la naissance > (Dessureault, 2015).

Heureusement, depuis les années 1960-1970, grâce aux mouvements féministes, les femmes réclament de plus en plus le retour aux pratiques moins interventionnistes, dans une optique où elles peuvent participer à la prise de décision relative à leur accouchement.

On ne pourrait pas passer sous silence le fait que le taux de mortalité infantile a diminué grandement, entre autres, en raison de l'utilisation de la procédure chirurgicale césarienne auprès des patientes à risque élevé, ce qui justifie l'intervention par les obstétriciens. Par ailleurs, la chirurgie devrait être soigneusement réservée aux parturientes vivant des complications lors de l'accouchement, afin de diminuer les risques ou sauver la vie des mamans et des bébés.

\section{Manque de ressources}

Dans la société actuelle, les Canadiens valorisent l'individualité et le respect des libertés. Dans ce même ordre d'idées, les futurs parents devraient être en mesure de pouvoir faire des choix libres et éclairés en matière de grossesse et d'accouchement. Or, on sait qu'à peine $2 \%$ des femmes accouchent naturellement avec l'aide d'une sage-femme, bien que le quart des Québécoises enceintes souhaiterait bénéficier de cette alternative (Dessureault, 2015). Le problème est causé par un manque de ressources dans notre réseau de la santé. Au Québec, on ne retrouve que seize maisons de naissances. Dans certaines régions du Québec, il y a des listes d'attente empêchant les femmes d'avoir recours à une sage-femme et dans d'autres régions, ce service n'est tout simplement pas présent (exemple : en AbitibiTémiscamingue, sur la Côte-Nord, en Gaspésie et dans la région de Laval), cependant les besoins sont grandissants.

Des 96 universités du grand territoire canadien, seulement 6 offrent la formation de sages-femmes; une au Québec, trois en Ontario (à l'intérieur d'un consortium formé des universités McMaster, Laurentienne et Ryerson), une à Calgary et une en Colombie-Britannique. Les programmes sont contingentés donc restreints à un certain nombre d'étudiants annuellement. De plus, il existe deux programmes d'appoint pour les sages-femmes formées à l'extérieur, afin qu'elles puissent pratiquer au Canada.

L'accès à la pratique des sages-femmes, et ce au niveau mondial, est restreint souvent dû à un manque de financement alors que contradictoirement, la pratique des sages-femmes génère moins de coût que la pratique des autres praticiens. Selon les auteurs Halem, Sandall, Devane, Soltani et Gates (2009), les décideurs qui désirent réaliser de grandes améliorations dans les soins de maternité, particulièrement sur la normalisation et l'humanisation de la 
naissance, devraient considérer les modèles de pratique de [sages-femmes] et envisager les moyens de les financer.

\section{Dépenses relatives à l'accouchement}

En 2015, Janssen, Mitton et Aghajanian conduisaient une recherche en Colombie-Britannique sur 9864 femmes qui répondaient aux critères de naissance à faible risque, pouvant donc toutes bénéficier des soins des sagesfemmes lors de leur accouchement (ces femmes présentaient des similitudes au niveau de l'âge, du statut parental, du revenu, de l'indice de masse corporelle et de la consommation de substances). À noter qu'en ColombieBritannique, $11 \%$ des accouchements sont actuellement assistés par des sagesfemmes. Parmi ceux-ci, environ $20 \%$ ont lieu en maison de sages-femmes (Janssen, Mitton et Aghajanian, 2015).

Les auteurs rapportent que les naissances à domicile avec une sagefemme sont moins dispendieuses pour le système de santé, et ce jusqu'à un an après la naissance du nouveau-né en comparaison avec les naissances en milieu hospitalier. Il en résulte d'une économie de $2338 \$$ pour les naissances dans un hôpital avec une sage-femme versus un accouchement à domicile et une économie de $2541 \$$ par rapport à un accouchement avec un médecin à l'hôpital, et ce, pour les 28 jours suivant l'accouchement (Janssen, Mitton et Aghajanian, 2015); pour les 8 semaines suivantes, une économie de $1683 \$$ par rapport à un accouchement à l'hôpital avec une sage-femme et de $1100 \$$ par rapport aux médecins. Pour l'année complète suivant la naissance, l'économie des coûts pour les nourrissons sont de $810 \$$ pour une naissance à l'hôpital avec une sage-femme et de $1146 \$$ d'économie pour une naissance avec un médecin (Janssen, Mitton et Aghajanian, 2015). Les coûts de transport sont le seul élément qui ne figure pas dans une économie de coût; pour le reste, c'est-àdire les honoraires de la sage-femme ou du médecin, les frais d'hospitalisation et le coût des pharmaceutiques, sont des éléments plus dispendieux pour le système de santé.

Ces résultats devraient encourager les femmes ne présentant que peu de risques durant leur grossesse à procéder à un accouchement à domicile et à promouvoir cette alternative pour les planificateurs et décideurs politiciens. Toutefois, le gouvernement de l'Ontario (Canada) a procédé à des restrictions budgétaires significatives touchant l'activité et la rétribution des sagesfemmes, malgré le signal d'alarme tiré par l'Organisation Mondiale de la Santé (OMS, 2013) à propos du besoin de sage-femmes et de l'importance de la reconnaissance des économies qu'elles apportent aux systèmes de santé dans le monde entier. 


\section{Réticences des médecins}

Dans certains cas, les médecins n'approuvent aucunement l'ouverture à la pratique des sages-femmes, restreignant ainsi les futures mères à faire confiance aux médecins, n'ayant pas ou peu d'autres options. Le contexte hospitalier et médical met peu en avant la liberté des patients, par souci d'efficacité et de rapidité de la part du personnel entraînant la standardisation des méthodes (Dessureault, 2015). Certains des médecins peuvent être réticents à l'entrée des sages-femmes dans le réseau, pour les responsabilités et les risques que cette démarche pourrait leur imposer. Ce serait au médecin de prendre le relais si des problèmes étaient rencontrés durant l'accouchement. La qualité du travail médical reposerait sur la qualité du travail de la sagefemme avant et au début de l'accouchement, cela impliquant une bonne relation de confiance entre le personnel médical et la sage-femme, ce que certains ne sont pas prêts à bâtir facilement.

Pourtant, les sages-femmes sont formées pour dépister un accouchement nécessitant des soins urgents d'un médecin ou spécialise et/ou d'une nécessité de transfert vers un centre hospitalier. De plus, elles sont formées pour stabiliser la mère et le bébé en attendant la prise en charge médicale hospitalière. Les raisons sont faibles pour le médecin de douter d'une sage-femme, puisqu'elles ont la formation nécessaire. Il est fort possible que les intérêts des corporations médicales canadiennes, soit des médecins obstétriciens, soient en opposition à l'émergence de la pratique des sagesfemmes qui risque de s'emparer des accouchements normaux et sans complications, ainsi voyant leur charge allégée des cas les plus 'faciles', conséquemment des revenus obtenus avec le moindre effort.

Enfin, si l'obstétricien et la sage-femme travaillaient en partenariat, le transfert des soins pourrait être facilité au besoin, car le médecin serait au courant de l'état de santé de la mère, du déroulement du travail et de l'accouchement, ainsi que des changements advenant pour la mère et le bébé. La relation de travail des deux professionnels de la santé serait mise au service du bien-être de la parturiente et du bébé, donc à la diminution des risques associés à toute naissance.

\section{Satisfaction des soins}

Les parturientes bénéficiant des services de sages-femmes sont plus satisfaites de leur expérience et de leurs soins lors d'un accouchement. En guise d'exemple, Halem, Sandall, Devane, Soltani et Gates (2009) ont étudié 12276 femmes dans quatre pays, selon un devis expérimental en onze essais. Leurs grossesses sont classées à bas risque obstétrical ou à risque mixte et ont reçus des soins en milieux hospitaliers et communautaires. Les résultats confirment que les femmes ayant reçu des soins des sages-femmes sont plus satisfaites des soins (par les explications, la préparation et le déroulement de 
l'accouchement et les perceptions des moyens pour gérer la douleur) que celles qui ont eu des soins des autres praticiens (médecins ou obstétricien).

\section{Les sages-femmes à l'étranger}

Il n'y a pas seulement au Canada que les sages-femmes subissent des barrières dans leur pratique. En 2014, des sages-femmes françaises ont fait la grève : elles déploraient un manque de reconnaissance de leur profession au sein de la société française et revendiquaient leur salaire et leur statut au sein de l'hôpital. Elles demandaient à être considérées comme praticiennes en milieu hospitalier, au même titre que les médecins. Les médecins contestent cet argument car ils considèrent que le travail des sages-femmes ne peut se comparer au leur.

$<<$ [les sages-femmes] ne sont pas assez considérées dans le système médical... Pourtant, dans notre pratique quotidienne, nous [les sages-femmes] faisons des gestes qui dépassent le cadre normal de notre exercice, ce qui arrange tout le monde, car on manque cruellement de personnel. Tout ce travail n'est pas reconnu : nous sommes les petites mains du système de santé>> (Belliard, 2014, p.1). Cependant, les sages-femmes, peu importe le pays où elles pratiquent, demandent une plus grande reconnaissance de leur profession.

\section{Conclusion}

La profession de Sage-femme mérite d'être plus promue, mieux valorisée et plus accessible au Canada, comme ailleurs. Compte tenu des difficultés budgétaires actuelles, le gouvernement aurait avantage à investir dans cette profession qui, de toute évidence, démontre clairement son efficacité dans la livraison des soins de qualité.

L'évolution et les progrès technologiques ont contribués à enrayer les pratiques traditionnelles et naturelles telles que l'accouchement à domicile ou en centre de naissances accompagné d'une sage-femme. Toutes les femmes n'ayant peu ou pas de risques de complication durant leur grossesse et désirant donner naissance sous la supervision d'une sage-femme devraient pouvoir bénéficier de cette option sécuritaire, tout simplement. Il serait plus humain, plus naturel, moins dispendieux et dégorgerait le système de santé et les obstétriciens.

\section{References:}

1. Belliard, D. (2013). Sage-femme, une profession invisible. Alternatives économiques, 333 (3). p. 28. Tiré de https://www.cairn.info/magazine-alternatives-economiques-2014-3p40.htm. Consulté en janvier 2019. 
2. Davies, B and Logan, J. (2011). Lire des textes de recherche. Milton, Canada : Elsevier

3. Dessureault, A-M. (2015). La médicalisation de l'accouchement : impacts possibles sur la santé mentale et physique des familles. Devenir, 27 (1), p. 53-68. DOI 10.3917/dev.151.0053

4. Hatem. M, Sandall. J, Devane. D, Soltani.H, Gates.S, (2009). Comparaison des modèles de soins obstétricaux dirigés par les sagesfemmes à d'autres modèles de soins offerts aux femmes enceintes : une revue systématique Cochrane. Pratiques et Organisation des Soins 40 (4) p. 267-274. DOI 10.3917/pos.404.0267

5. Janssen P.A, Mitton. C, Aghajanian. J. (2015). Costs of planned home vs. hospital birth in british-columbia attended by registered midwives and physicians. PLoS ONE 10(7). P.1-11. DOI:10.1371/journal.pone.0133524

6. MacDonald, M., E. (2018). The Making of Informed Choice in Midwifery: A Feminist Experiment in Care. Culture, Medecine and Psychiatry 42. P.278-294 DOI: 10.1007/s11013-017-9560-9

7. McCracken, K. (2015). A Patchwork of Care : Midwifery in Canada. Tiré de http://activehistory.ca/2015/01/a-patchwork-of-caremidwifery-in-canada/, le 28 décembre 2018.

8. Mélanson, N. (1999). L'évolution de la profession de sagefemme. Reflets 5(2), p.247-255. DOI :10.7202/026283ar

9. Morgan. L, Carson. G, Gagnon. A et Blake, J. (2014). Collaborative practice among obstetricians, family physicians and midwives, CMJA 186(17). P.1279 et 1280. DOI:10.1503/cmaj.140537/-/DC1

10. Organisation Mondiale de la Santé (OMS) (2013). Il faut davantage de sages-femmes pour améliorer la survie de la mère et du nouveau-né. Bulletin de l'Organisation mondiale de la Santé 2013 ; 91 : 804-805.

a. doi: http://dx.doi.org/10.2471/BLT.13.021113.

11. Wagner. M. (2016). Global Midwifery and the Humanization of Birth. Midwifery today, 39 (4). p.39-40. Tiré de https://midwiferytoday.com/mt-articles/globalmidwiferyhumanization-birth/ . Consulté en janvier 2019. 